\title{
Analysis and Design of E-Commerce Information Systems Using the User Centered Design Method at PT. Pure Healthy Nature
}

\author{
Amelia Andi', Yakub ${ }^{2}$ \\ ${ }^{1,2}$ Information System, Buddhi Dharma University, Banten, Indonesia
}

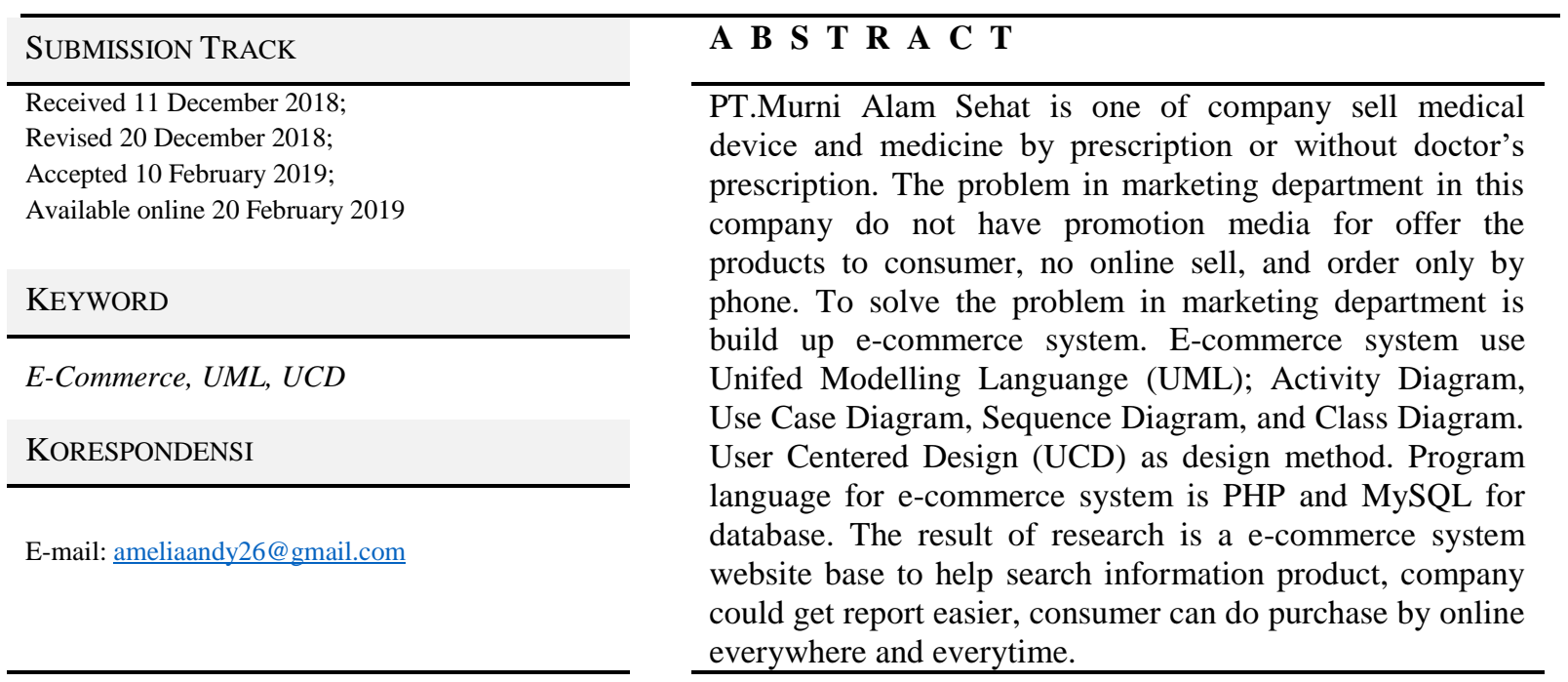

\section{INTRODUCTION}

As time goes by technology, especially the Internet, now the reach between buyers and sellers is extensive, all time constraints, distances, and costs can be resolved easily. One of the types of technology implementation in terms of improving business, sales and purchasing products is to use e-commerce [1]. PT. Murni Alam Sehat is a company supplies medical devices and medicines by a recipt orgeneric type. The problems that occur in PT. Murni Alam Sehat are in the sales. Issues that occur among others ; (1) The company do not have promotional media to offer their products to consumers. (2) Do not have E-commerce system, so consumers must go to company to buy product. (3) The order process is by telephone only and directly. Based on the problems that are needed in the e-commerce system to assist the company's business processes designed with Unified Modeling Language (Activity Diagram, Use Case Diagram, Sequence Diagram, Class Diagram). Program language used in making this information system is PHP, MySQL database, and User Centered Design method. This system is expected to facilitate consumers in ordering products quickly, consumers can access the information needed at any time and anywhere without having to come directly to the company, facilitate the company in the activities Business such as sales and promotion of new products, helping the company's business process become faster and expanding its marketing reach. 


\section{LITERATURES REVIEW}

This Research of User Centered Design is familiar, one of them research by [2]. In the research explained the difficulties of current users is that the company still use the conventional sales system, where the customer directly to the company to make the purchase of the product. With the conventional system often the company is experiencing problems such as limited reach of purchasing to customers who are outside the city. This is because information about the company's sales is not yet accessible to all customers. Then the solution of the problem is solved by designing an ecommerce website using the User Centered Design method by involving the participation of users into its design. The methods used in system development use Questionnaires and usability testing.

\section{FRAMEWORK}

Here is the framework created:

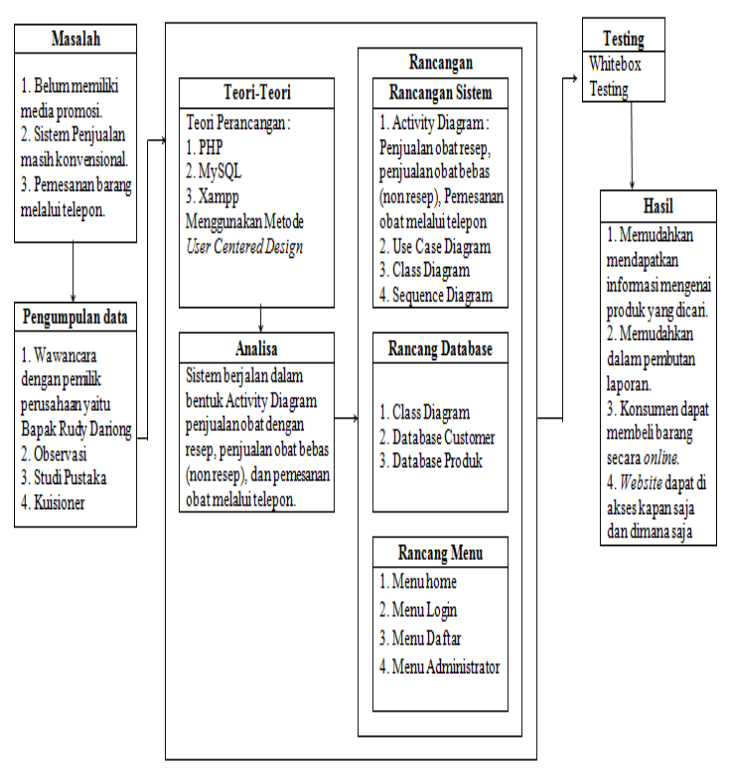

Figure 1. Framework

\section{METHODS}

\section{E-Commerce}

E-Commerce is the dissemination, purchase, sale, marketing of goods and services by electronic systems such as internet or television, WWW (World Wide Web), or other computer networks. E-commerce involves electronic funds transfer, electronic data exchange, automated inventory management system, and automatic data collection system [3].

E-commerce has benefits and advantages for various parties, among others [4] :

1. For the company:
a. National and international market availability
b. Decrease costs for processing, distribution, and withdrawal of information

2. For Community

a. Can access products and services, 24 hours a day

b. Providing information services easily and comfortably to consumers

\section{User Centered Design}

User Centered Design (UCD) is a new paradigm in the development of web-based systems. The concept of UCD is the user as the center of the system development process, objectives, context and system environment are all based off the user experience [5].

The process in User Centered Design is [6]:
a. UCD Planning
b. Understand and determine user context
c. Determine user needs
d. The resulting design solutions
e. Evaluation of design to user needs

\section{Website}

Website is a collection of pages used to display text, image, animation, sound, or 
combination of all, both static and dynamic, forming a series of interrelated buildings, and each of them connected by a network of pages [7].

The website is divided into 2 groups, among others [8]:

a. Static Website

b. Dynamic Website

\section{RESULT}

\section{User Requirements Analysis}

In this step is carried out the distribution of the questionnaire to 15 respondents to know user needs for features and visual design such as what is needed and liked by the user. The questionnaire that was spread before the construction of this system consisted of 10 numbers of questions, such as :

Table 1. User Requirements Analysis Results

\begin{tabular}{|c|c|c|c|c|c|c|}
\hline No & Pertanyaan & 1 & 2 & 3 & 4 & 5 \\
\hline 1 & $\begin{array}{l}\text { Apakah aplikasi mengenai pembelian obat secara } \\
\text { online harus memberikan informasi secara lengkap } \\
\text { dan detail? }\end{array}$ & & & 2 & 7 & 6 \\
\hline 2 & $\begin{array}{l}\text { Apakah aplikasi mengenai pembelian obat secara } \\
\text { online perlu mengelompokkan obat berdasarkan } \\
\text { jenis penyakit? }\end{array}$ & & & 1 & 7 & 7 \\
\hline 3 & $\begin{array}{l}\text { Apakah keterangan mengenai obat perlu } \\
\text { ditampilkan dengan penjelas an yang cukup lengkap } \\
\text { disertai dengan gambar produk? }\end{array}$ & & & 2 & 7 & 6 \\
\hline 4 & $\begin{array}{l}\text { Menurut saudara, perlukah menampikan form } \\
\text { pencarian pada website? }\end{array}$ & & & & 8 & 7 \\
\hline 5 & $\begin{array}{l}\text { Apakah tampilan daftar produk pada website } \\
\text { ditampilkan dalam bentuk grid? }\end{array}$ & & 1 & 3 & 5 & 6 \\
\hline 6 & $\begin{array}{l}\text { Apakah tampilan daftar produk pada website } \\
\text { ditampilkan dalam bentuk list? }\end{array}$ & & 4 & 6 & 3 & 2 \\
\hline 7 & $\begin{array}{l}\text { Apakah warna yang cerah lebih menarik untuk } \\
\text { tampilan website? }\end{array}$ & & 2 & 2 & 5 & 6 \\
\hline 8 & $\begin{array}{l}\text { Apaksh perlu menampilkan promo produk pada } \\
\text { halaman depan? }\end{array}$ & & 2 & 3 & 5 & 5 \\
\hline 9 & $\begin{array}{l}\text { Apakah penggunaaan bahasa Indonesia lebih baik } \\
\text { digunakan pada website? }\end{array}$ & & 2 & 2 & 3 & 8 \\
\hline 10 & $\begin{array}{l}\text { Apakah letak form login dan register sebaiknya } \\
\text { ditempatkan di bagian atas? }\end{array}$ & & 2 & 3 & 4 & 6 \\
\hline & Total & 0 & 13 & 24 & 54 & 59 \\
\hline
\end{tabular}

Data obtained from the respondent can be known percentage for each assessment as follows :

Strongly disagree $=(0 / 150) \times 100 \%=0$
Disagree $=(13 / 150) \times 100 \%=8.67 \%$

Neutral $=(24 / 150) \times 100 \%=16 \%$

Agree $=(54 / 150) \times 100 \%=36 \%$

Strongly agree $=(59 / 150) \times 100 \%=39.33 \%$

\section{Usability Value testing (Usability Testing)}

The following is a usability testing conducted using the Likert scale calculation method :

$$
\% \text { score }=\frac{\text { actualscore }}{\text { idealscore }} \times 100 \%
$$

After get the result in the quantitative value from calculation, then the value is converted to a qualitative value of 5 with the reference of Likert scale as in:

Table 2. Percentage interval

\begin{tabular}{|c|c|c|}
\hline No & $\%$ & Kriteria \\
\hline 1 & $0,00-20,00$ & Tidak Baik (1) \\
\hline 2 & $20,01-52,00$ & Kurang Baik (2) \\
\hline 3 & $52,01-68,00$ & Cukup Baik (3) \\
\hline 4 & $68,01-84,00$ & Baik (4) \\
\hline 5 & $84,01-100$ & Sangat Baik (5) \\
\hline
\end{tabular}

Usability Valuation Testing was conducted using the usability testing questionnaire completed by 15 respondents. The following is the data obtained from usability test.

\begin{tabular}{|c|c|c|c|c|c|c|}
\hline No & Pertanyaan & 1 & 2 & 3 & 4 & 5 \\
\hline \multicolumn{7}{|c|}{ A. Mudah di pelajari (Learnability) } \\
\hline 1 & $\begin{array}{l}\text { Apakah tulisan yang digunakan } \\
\text { pada halaman utama dapat di baca? }\end{array}$ & & & 5 & 5 & 5 \\
\hline 2 & $\begin{array}{l}\text { Apakah menu yang ada sudah } \\
\text { lengkap serta mudah dipahami dan } \\
\text { dimengerti? }\end{array}$ & & & 6 & 4 & 5 \\
\hline 3 & $\begin{array}{l}\text { Apakah secara visual pengguna } \\
\text { dapat mengerti kegunaan tiap } \\
\text { tombol yang disediakan pada } \\
\text { commerce ini? }\end{array}$ & & & 6 & 5 & 4 \\
\hline 4 & $\begin{array}{l}\text { Apakah sistem dapat memberikan } \\
\text { tahap-tahap penggunaan sistem } \\
\text { (user manual) }\end{array}$ & & 2 & 4 & 5 & 4 \\
\hline \multicolumn{7}{|c|}{ B. Efisiensi (Efficiency) } \\
\hline 5 & $\begin{array}{l}\text { Apakah pergantian satu halaman ke } \\
\text { halaman lainnya tidak } \\
\text { membutuhkan waktu yang lama? }\end{array}$ & & & 2 & 7 & 6 \\
\hline 6 & $\begin{array}{l}\text { Apakah sistem tidak mengandung } \\
\text { halaman yang tidak dibutuhkan } \\
\text { pengguna? }\end{array}$ & & 2 & 3 & 5 & 5 \\
\hline 7 & $\begin{array}{l}\text { Apakah saat diketikan pada form } \\
\text { pencarian produk dapat } \\
\text { ditampilkan? }\end{array}$ & & & 3 & 6 & 6 \\
\hline
\end{tabular}

Table 3. Usability Value Results 


\begin{tabular}{|c|l|l|l|l|l|l|}
\hline \multicolumn{2}{|c|}{ C. Mudah Diingat (Memorability) } & & & & & \\
\hline 8 & $\begin{array}{l}\text { Apakah tampilan halaman website } \\
\text { mudah dingat? }\end{array}$ & & & 3 & 7 & 5 \\
\hline 9 & $\begin{array}{l}\text { Apakah, warna latar belakang } \\
\text { website menarik dan sesuai dengan } \\
\text { visualisasi pengguna? }\end{array}$ & & & 4 & 6 & 5 \\
\hline 10 & $\begin{array}{l}\text { Apakah ditemukan link sistem } \\
\text { yang salah atau emor ketika di } \\
\text { klik? }\end{array}$ & & & 6 & 4 & 5 \\
\hline 11 & $\begin{array}{l}\text { Apakah ada halaman bantuan pada } \\
\text { 5aat melakukan pembelian produk? }\end{array}$ & & 2 & 5 & 4 & 4 \\
\hline \multicolumn{2}{|c|}{ E. Kepuasan (Satisfaction) } & & & & & \\
\hline 12 & $\begin{array}{l}\text { Apakah informasi mengenai detail } \\
\text { produk disajikan secara lengkap? }\end{array}$ & & 1 & 3 & 6 & 5 \\
\hline 13 & $\begin{array}{l}\text { Apakah gambar dari produk di } \\
\text { website ditampilkan dengan jelas? }\end{array}$ & & & 3 & 5 & 7 \\
\hline 14 & $\begin{array}{l}\text { Apakah saudara merasa nyaman } \\
\text { menggunakan aplikasi website ini? }\end{array}$ & & & 4 & 7 & 4 \\
\hline 15 & $\begin{array}{l}\text { Apakah saudara ingin mengunjungi } \\
\text { kembali halaman website e- } \\
\text { commerce PT. Murni Alam Sehat? }\end{array}$ & & 5 & 6 & 4 \\
\hline
\end{tabular}

Table 4. Analysis of Test Data

\begin{tabular}{|c|c|c|c|}
\hline $\begin{array}{c}\text { Pertanyaan } \\
\text { No }\end{array}$ & Skor Total & Skor Maksimal & Presentase \\
\hline 1 & 60 & 75 & $80,00 \%$ \\
\hline 2 & 59 & 75 & $78,66 \%$ \\
\hline 3 & 58 & 75 & $77,33 \%$ \\
\hline 4 & 56 & 75 & $74,66 \%$ \\
\hline 5 & 64 & 75 & $85,33 \%$ \\
\hline 6 & 58 & 75 & $73,33 \%$ \\
\hline 7 & 63 & 75 & $84,00 \%$ \\
\hline 8 & 62 & 75 & $82,66 \%$ \\
\hline 9 & 61 & 75 & $81,33 \%$ \\
\hline 10 & 59 & 75 & $78,66 \%$ \\
\hline 11 & 55 & 75 & $73,33 \%$ \\
\hline 12 & 60 & 75 & $80,00 \%$ \\
\hline 13 & 64 & 75 & $85,33 \%$ \\
\hline 14 & 60 & 75 & $80,00 \%$ \\
\hline 15 & 59 & 75 & $78,66 \%$ \\
\hline Total & $\mathbf{8 9 8}$ & $\mathbf{1 1 2 5}$ & $79,02 \%$ \\
\hline
\end{tabular}

Calculation result from table 4 as follows:

Percentage of usability value $=889 / 1125 \mathrm{x}$ $100 \%$

Percentage of usability value $=889 / 1125 \mathrm{x}$ 100

Percentage of usability value $=79.02 \%$ Based on the calculation of usability value data, the system has usability value of $79.02 \%$ and has a good scale.

\section{System Testing}

To find out the results of a website ecommerce system, then disseminated the questionnaire to collect data. From the results of the questionnaire we can see the respondents response to the e-commerce system created.
Here are the 3 questions selected as a system test result:

Table 5. System Test Results

\begin{tabular}{|c|l|c|c|}
\hline No & \multicolumn{1}{|c|}{ Pertanyaan } & Ya & Tidak \\
\hline 1 & $\begin{array}{l}\text { Apakah dengan adanya sistem e-commerce } \\
\text { mendapatkan informasi mengenai produk menjadi } \\
\text { lebih mudah? }\end{array}$ & 5 & 0 \\
\hline 2 & $\begin{array}{l}\text { Apakah sistem e-commerce ini mudah digunakan? } \\
3\end{array}$ & $\begin{array}{l}\text { Apakah dengan adanya sistem e-commerce dapat } \\
\text { memudahkan saudara melakukan pembelian online? }\end{array}$ & 4 \\
\hline
\end{tabular}

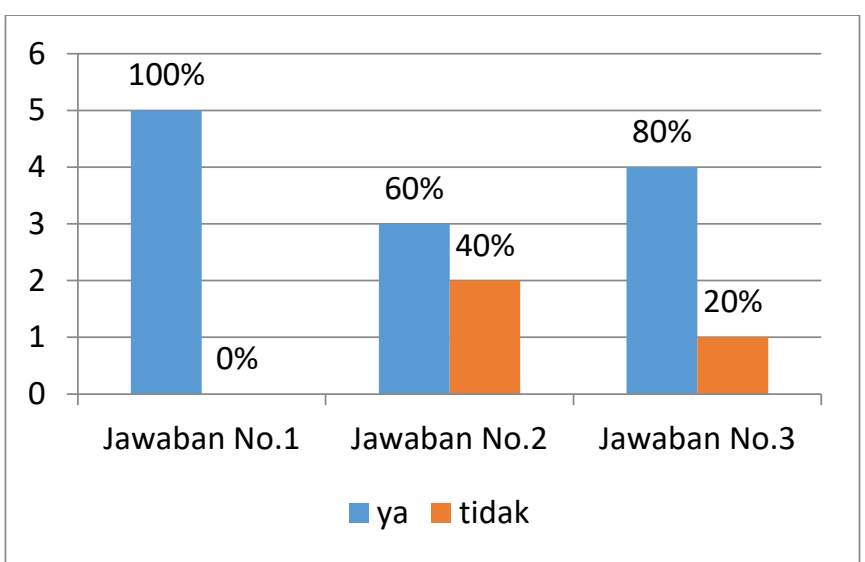

Figure 2. System test Results Diagram

From the whole questionnaire, it was concluded that all respondents agreed that this e-commerce system can provide information about the product more easily, $60 \%$ of respondents agreed that the ecommerce system is easy to use, and $80 \%$ of respondents Agree that this e-commerce system can facilitate the online purchase process.

\section{DISCUSSION}

\section{Design Analysis Using Activity Diagrams}

Activity Diagram used to proposed system illustrates to make it easier and understand the proposed system. 


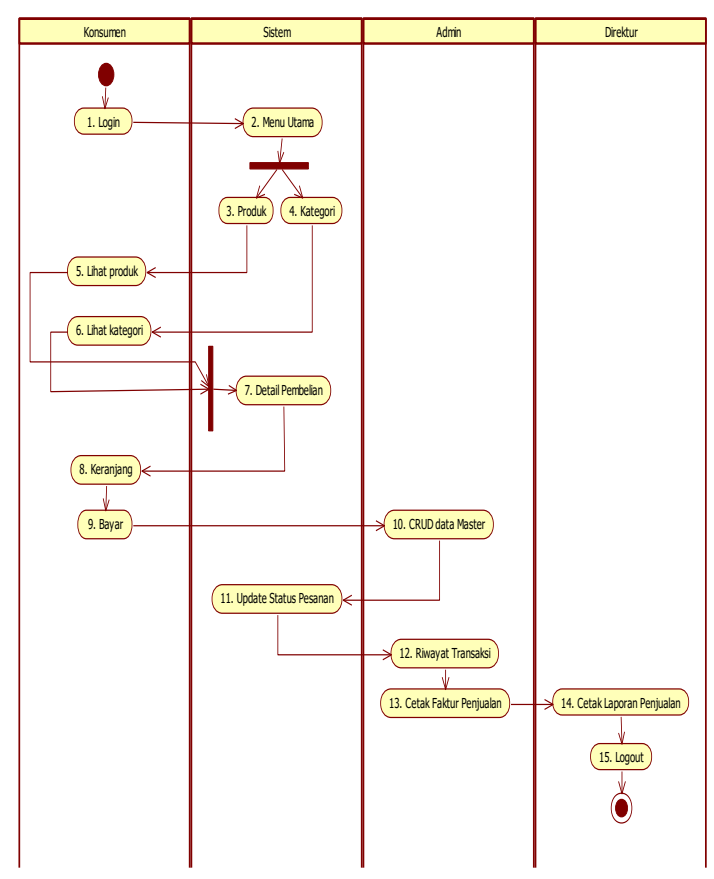

Figure 3. Activity Diagram

\section{Use Case Diagram}

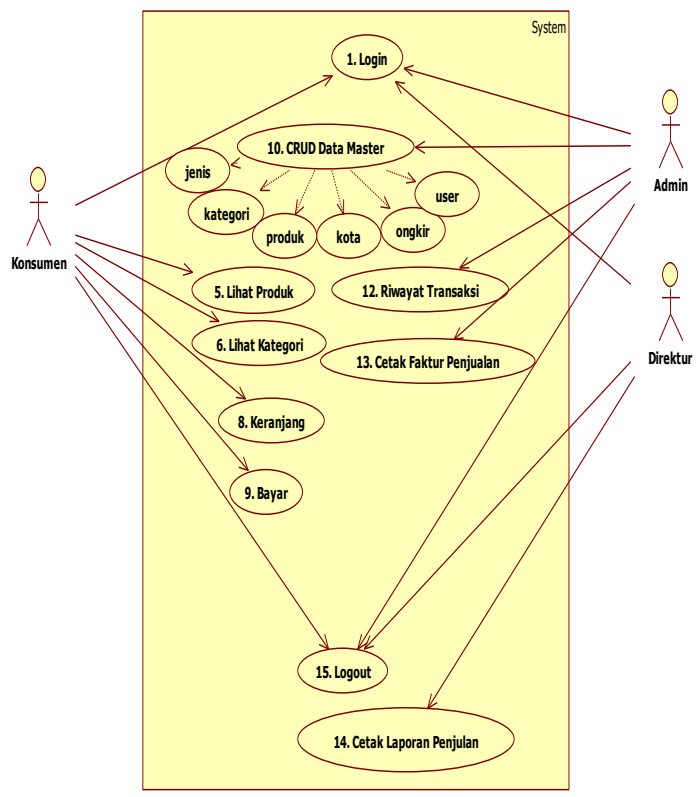

Figure 4. Use Case Diagram

\section{Sequence Diagram}

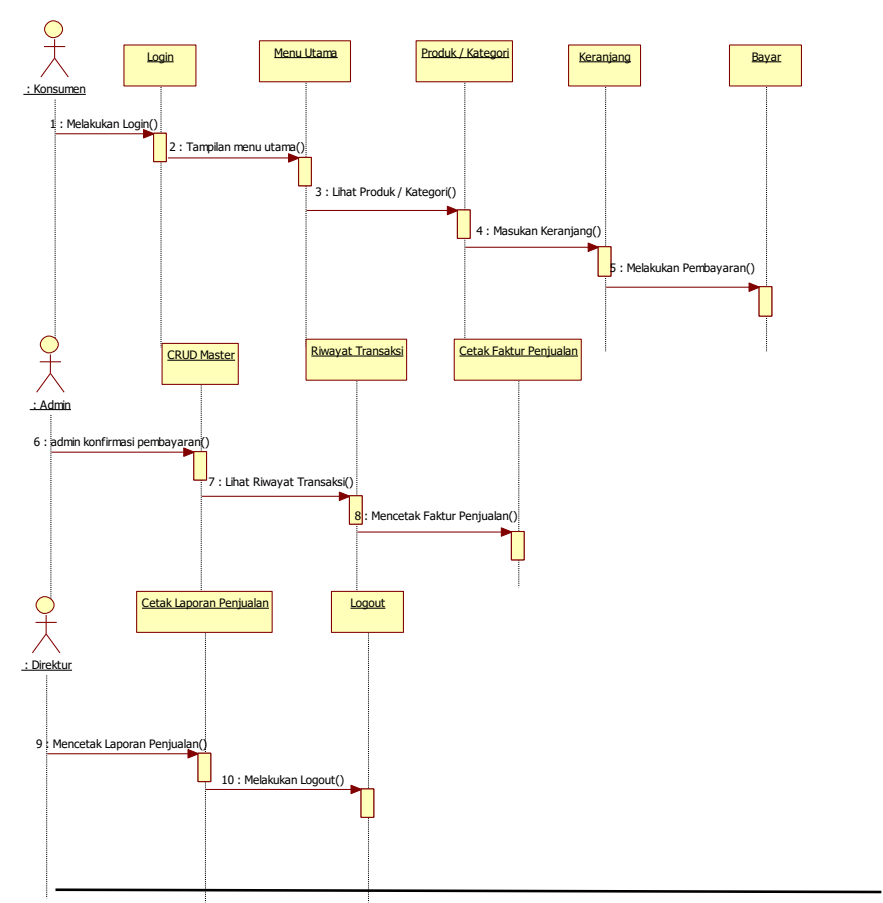

Figure 5. Sequence Diagram

\section{Class Diagram}

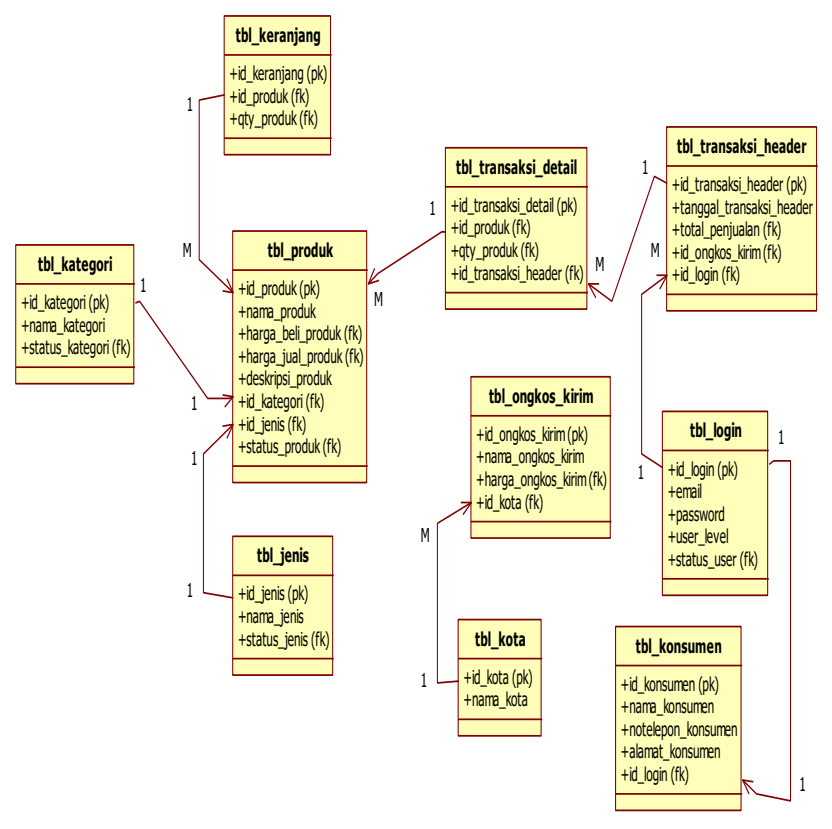

Figure 6. Class Diagram

\section{User Interface}

Here are some of the user interfaces / views of website have been created or implemented : 


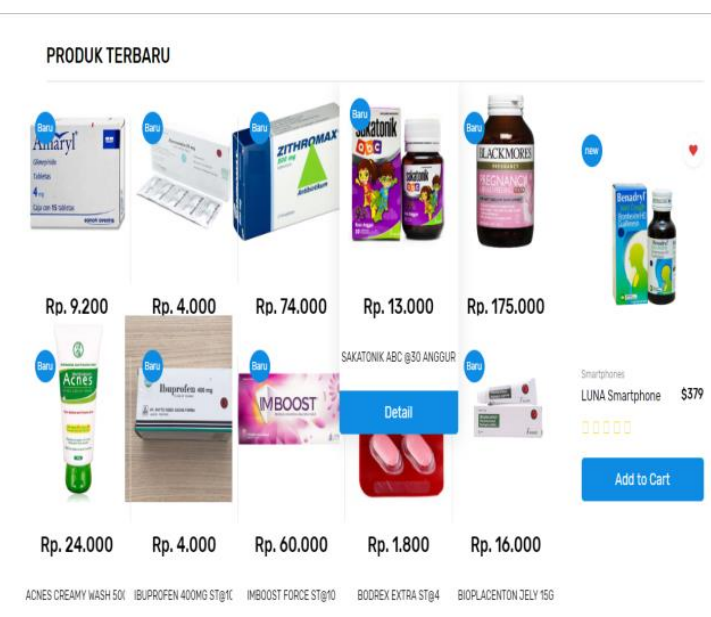

Figure 7. Product View

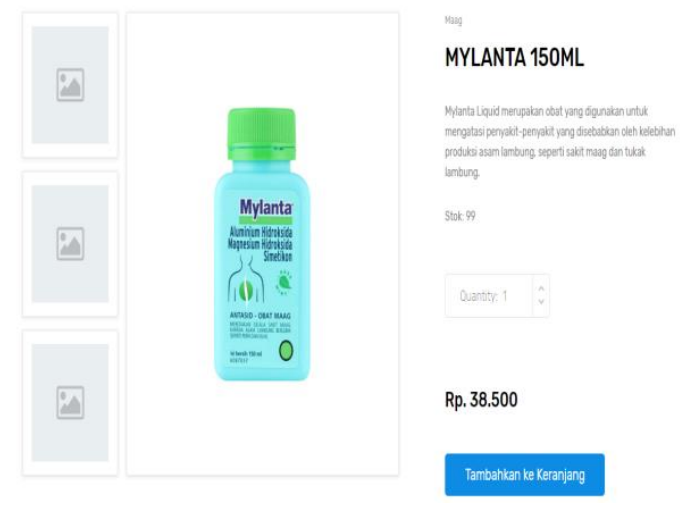

Figure 8. Product Details

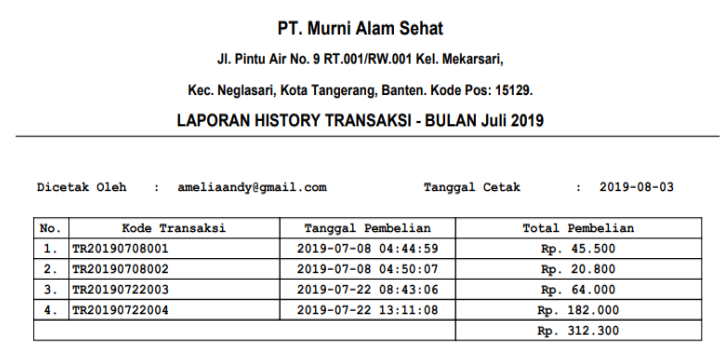

Figure 9. History Transactions Report

\section{CONCLUSION}

After research and observation at PT. Murni Alam Sehat can be concluded as follows:

a. Based on system testing, all respondents agreed the e-commerce system made it easy consumers to get information about the products they are making quickly and easily without come to the company.

b. This e-commerce system makes it easy to create a purchase transaction history report because it can automatically be printed according to company needs.

c. Based on a system testing conducted as many as $80 \%$ of respondents agree with ecommerce system, consumers can buy product by online system.

d. Based on a system testing as many as $60 \%$ of respondents agree this e-commerce system is easy to use and can be accessed anytime and anywhere.

\section{REFERENCES}

[1] Hidayat, Taufik. 2008, Panduan Membuat Toko Online dengan OS Commerce. Jakarta: Media Kita.

[2] Intan Sandra Yatana Saputri, dkk, "Penerapan Metode UCD (User Centered Design) pada E-Commerce Putri Intan Shop Berbasisi Web", Jurnal Teknologi Sistem Informasi, Vol. 03, 2017, pp.4-8.

[3] Sutabri, Tata. 2012, Konsep Sistem Informasi. Yogyakarta : Andi. 
[4] Turban, David K., et. al. 2012. Electronic Commerce 7th Edition. United States : Pearson.

[5] Simatupang, R. M. 2014. Penerapan Metode UCD untuk Perancangan Aplikasi Radio Streaming Berbasis Web. Informasi Dan Teknologi Ilmiah (INTI), III.

[6] Albani, L., \& Lombardi, G. (2011). User Centred Design for EASYREACH.

[7] Bekti, Bintu Humairah. 2015, Mahir Membuat Website dengan Adobe Dreamweaver CS6, CSS dan JQuery. Yogyakarta: Andi.

[8] Santosa, Ippho. 2012, 7 Keajaiban Rezeki. Jakarta : Gramedia.

\section{BIOGRAPHY}

Amelia Andi, Graduated from the Information Systems Study Program (S1) in the academic concentration of Information Technology at 2019.

Yakub, Lecturer at Information System Study Program of Buddhi Dharma University, Tangerang. Graduates S1 Information System STMIK Widuri Jakarta, 2002, S2 Master Computer Eresha School of Information Technology, 2005, S2 Master of Management of Budi Luhur University, 2008, S3 Doctoral University of Islam Nusantara Bandung, 2014. 